\title{
Investigation of circulation noise effect on SAC-OCDMA system with ring topology
}

\begin{abstract}
In this paper, we investigate the performance of an OCDMA system with ring topology where number of the spans can be more than one. Effect of circulation noise is evaluated beside of shot noise and thermal noise. Through analytical approach, it is shown that higher amount of amplifier gain, produces higher amount of circulation noise. Also by increasing the number of nodes, the strength of the circulation noise is reduced and the ratio of this reduction is more for lower amplifier gain. For example, with increasing number of the nodes from 3 to 8 , the amount of $\log$ variance of circulation noise increases only 1 unit (-13 to -14$)$ for amplifier gain $20 \mathrm{dBm}$, while this value is 4 units (from -15 to -19 ) if gain is $10 \mathrm{dBm}$.
\end{abstract}

Keyword: Circulation noise; Optical code division multiple access (OCDMA); Optical networks; Ring topology; Spectral amplitude coding (SAC) 